\title{
LA GEOGRAFÍA EN \\ LA HISTORIOGRAFÍA HELENÍSTICA. \\ EL CONCEPTO DE OIKOUMENE EN LAS \\ HISTORIAS DE POLIBIO
}

\author{
Leslie Lagos-Aburto \\ Felipe Montanares-Piña \\ Universidad de Concepción. Chile
}

\begin{abstract}
Resumen: El desarrollo de la geografía durante el período helenístico, por un lado, estuvo determinado por las conquistas de Alejandro que expandieron los horizontes de la oikoumene hacia territorios desconocidos para el mundo griego, y por otro, por una tradición geográfica que relacionó elementos de otras disciplinas, tales como: la literatura, la filosofía, la techné y la historia. Tradicionalmente el concepto de oikoumene se entiende como el espacio donde se expande y desarrolla la cultura griega. A lo largo del siglo II a.C., en su búsqueda de dominio universal, Roma logró dominar políticamente el espacio griego, pero integrándose a su vez a la oikoumene griega. El presente trabajo propone considerar que la historiografía helenística utilizó recursos de la tradición geográfica griega para lograr configurar un nuevo espacio, compuesto por el mundo greco-helenístico y los territorios incorporados a dominio romanos de la cuenca mediterránea occidental. Para ello se realizará un análisis de las Historias de Polibio, obra que con un objetivo universalista, integró los elementos de la geografía helenística para construir un nuevo concepto de oikoumene.
\end{abstract}

Palabras claves: Oikoumene - Geografía - Polibio - Historiografía helenística. 


\title{
THE GEOGRAPHY IN HELLENISTIC HISTORIOGRAPHY. THE CONCEPT OF OIKOUMENE IN THE HISTORIES OF POLYBIUS
}

\begin{abstract}
The development of geography during the hellenistic period, on one hand, was determined by the conquests of Alexander that expanded the horizons of the oikoumene towards territories unknown to the Greek world, and on the other, by a geographical tradition that related elements of other disciplines, such as: literature, philosophy, techné and history. Traditionally the concept of oikoumene is understood as the space where the greek culture expands and develops. Throughout the II century BC, in its search for universal dominion, Rome managed to dominate the Greek space politically, but integrated itself into the greek oikoume$n e$. The present work proposes to consider that the hellenistic historiography used resources of the greek geographical tradition to manage to configure a new space, composed by the greco-hellenistic world and the territories incorporated to Roman domain of the western Mediterranean basin. For this, an analysis of the Histories of Polybius will be carried out, a work that, with a universalist objective, integrated the elements of hellenistic geography to construct a new oikoumene concept.
\end{abstract}

Keywords: Oikoumene - Geography - Polybius - Hellenistic historiography.

Recibido: 10.09.2019 - Aceptado: 31.03.2020

\section{Correspondencia:}

\section{Leslie Lagos-Aburto}

Correo electrónico: 1lagos@udec.cl

Doctora en Historia por la P. Universidad Católica de Valparaíso. Profesora de Historia Antigua del Departamento de Historia de la Universidad de Concepción. Miembro del Grupo de Estudios Interuniversitario del Mediterráneo Antiguo (GEIMA).

ID Orcid: https://orcid.org/0000-0002-9210-8056

\section{Felipe Montanares-Piña}

Correo electrónico: felimontanares@udec.cl

Licenciado en Historia y candidato a Magister en Historia por la Universidad de Concepción. Becario de la Agencia Nacional de Investigación y Desarrollo (ANID) / Programa de Becas / Beca Magíster Nacional 2020 / 22200082.

ID Orcid: https://orcid.org/0000-0001-7627-5682 


\section{Introducción ${ }^{1}$}

T a historiografía del período helenístico para autores como A. Momigliano, T. Scanlon, K. Meister y M. Betalli se expandió en la medida que se propagó la cultura griega a través de diferentes procesos desde las conquistas de Alejandro hasta la caída de los reinos helenísticos ${ }^{2}$.

El concepto a analizar en este trabajo es la idea griega de oikoumene, definido por Antonio Molina como el espacio o mundo habitado, pero civilizado $^{3}$. Esta explicación se realiza desde una perspectiva cultural, lo que permite determinar que en la medida en que la cultura griega se propaga, el espacio griego civilizado también lo hace, incluyendo nuevos territorios dentro de la oikoumene, y además, se debe comprender no solamente este término como el mundo habitado, sino que de igual forma, como el espacio civilizado.

Igualmente se puede percibir este concepto desde una perspectiva política como lo presenta María José Hidalgo de la Vega y Gonzalo Cruz Andreotti, quienes advierten que este término se encuentra inserto en una serie de temas y aspectos de gran complejidad que se modifican y vuelven a articularse en la medida que el pensamiento griego evolucionó, tomando de este variados matices, pero asimismo, destacan la importancia de oikoumene en los procesos que implicaron anexión y conquista de territorios, pueblos y sociedades bajo la idea de comunidad, involucrando una naturaleza integradora y al mismo tiempo excluyente hacia lo que es diferente. Esto último se expresó en actitudes complejas y contradictorias hacia los bárbaros conquistados y no conquistados, obteniendo por consecuencia la exclusión, discriminación y demonización de las diversas formas de alteridades ${ }^{4}$.

Indicamos, por otra parte, una tendencia en los trabajos realizados en los últimos años que llevan a concebir el concepto de oikoumene como

1 Este estudio está inserto en el Proyecto de Iniciación a la Investigación de la Vicerrectoría de Investigación y Desarrollo de la Universidad de Concepción, Chile, código 217.064.045-OIN.

MOMIGLIANO (1984: 25-30); SCANLON (2015: 277-279); MEISTER (1992: 91-ss); BETTALLI (2009).

MOLINA (2011:29-30).

HIDALGO DE LA VEGA. (2005); CRUZ, G. (2004). 
un equivalente al orbe romano, no obstante, para el desarrollo de nuestro trabajo esto no se debe comprender de esta manera. Principalmente se suele asumir que desde la batalla de Pidna (167 a.C.) el concepto de oikoumene pasó a ser una categoría equivalente a los territorios donde Roma ejerció su dominio. Este control ecuménico se proyectó durante el último período de la República, y fue en la época del principado, sobretodo en el siglo II, donde se veló en estricto rigor por la protección de los ideales de la oikoumene. ${ }^{5}$

El desarrollo de la oikoumene se relaciona con la evolución de la concepción griega del espacio en la medida que las coyunturas lo permiten. Esto nos faculta a pensar que el espacio griego estuvo delimitado con relación a la expansión cultural griega y en donde se encuentre presente la paideía se hallará lo griego, por tanto, la civilización formará parte de la oikoumene ${ }^{6}$. En este sentido, los siguientes autores: Domingo Placido ${ }^{7}$, Gonzalo Cruz Andreotti ${ }^{8}$, María José Hidalgo de la Vega ${ }^{9}$ y Claude Nicolet ${ }^{10}$ coinciden en señalar que la oikoumene es un concepto griego que ante la dominación romana fue utilizado para configurar una nueva realidad espacial que se acomodaba a las necesidades de dominio imperial (en sentido territorial), remitiéndose principalmente a Polibio y Estrabón para justificar tales planteamientos.

El desarrollo de las ciencias en la antigüedad se caracterizó por poseer un carácter integrador entre las diferentes disciplinas. Los griegos no tenían una clara división de sus objetos de estudios, tanto la mitología, la literatura, las matemáticas, la filosofía y la geografía, entre otras disciplinas, se integraron bajo un concepto, el de techné. ${ }^{11}$ Esta palabra envuelve una amplia cantidad de campos de estudios, dentro de los que se

$5 \quad$ HIDALGO DE LA VEGA. $(2005,2006)$.

6 BURSTEIN. (2016:59-78).

$7 \quad$ Pese a que reconoce el origen griego del término, lo relaciona con las ideas de integración romana, PLÁCIDO, D (2008, 2008ª 1997).

8 CRUZ ANDREOTTI $(2014,2009,2004,2002)$.

9 HIDALGO DE LA VEGA (2008, 2006, 2005); CARBO \& HIDALGO DE LA VEGA. (2008).

10 NICOLET $(2015,1989,1982)$.

11 MOLINA (2011:31). 
encuentran la geografía y la historia, de modo que debemos establecer cuál es la relevancia de la techné en la formación del pensamiento geográfico y que insertará el concepto de oikoumene dentro del relato historiográfico.

El concepto de techné tiene diferentes naturalezas. Podemos comprenderlo según la idea de Robert Meagher, quien señala que para el mundo griego implicó la producción de ideas. ${ }^{12}$ A esta reflexión debemos añadir la desarrollada por Richard Parry, quien indica que se debe reconocer una división entre las ideas de episteme, entendida como el dominio del conocimiento, y la techné, concebida como el desarrollo de la práctica. Estos son los elementos que van a constituir la relación dicotómica entre la evolución del pensamiento y el desarrollo de la técnica en la antigüedad. ${ }^{13}$ Por lo anterior, podemos deducir que la palabra techné fue utilizada por los griegos para definir todo el proceso que se inicia con el planteamiento de cuestiones y problemas, hasta la producción física de estas. ${ }^{14}$

Desde la techné se desarrollaron diversos campos de estudio, siendo uno de estos la geografía, que en un comienzo tuvo una gran cantidad de nombres para designarla, y además de abordar muchos temas, desde lo político a lo literario por ejemplo, pero siempre teniendo un propósito perceptible: el definir la relación entre el espacio y el hombre. ${ }^{15}$

En el caso particular del mundo griego, la geografía y la historia nacieron relacionadas, ya que la historia dota de un elemento temporal a la acción geográfica de analizar los elementos físicos del espacio. De igual manera, la historia se relaciona con otros campos de estudios pertinentes al desarrollo geográfico, como es el caso de la etnografía, la astronomía y las ciencias naturales. ${ }^{16}$ Es por ello que se hace necesario para nuestra investigación comprender el concepto que los griegos utilizaron para poder representar su espacio, cómo fue comprendido culturalmente por ellos y finalmente, cuál fue la proyección que tuvo a través del tiempo.

El término que los griegos utilizaron para representar su espacio recibió el nombre de oikoumene, y este fue un elemento de importancia para

12 Entendidas como un proyecto, planificación o teoría. MEAGHER (1988:158-159)

TABACHNICK (2004:90); PARRY (2003).

14 TUPLIN, RIHLL \& WOLPERT (2002:305).

15 MOLINA (2011:18).

KAPLAN (2018:195). 
el desarrollo de la historiografía helenística, ya que favoreció a estructurar el espacio territorial de las obras históricas, funcionando como una herramienta que permitió un enfoque holístico en los temas que se buscaba tratar, substancialmente cuando esta tendió a un carácter "universal", es decir, en la búsqueda de abarcar la totalidad.

\section{Oikumene expresión del espacio griego}

El concepto oikoumene fue utilizado por el mundo griego para determinar el espacio habitado. Según Klaus Geus, no debe entenderse solamente como una categoría religiosa o cultural, este debe pensarse como una categoría geográfica en el que los helenos representaron el mundo habitado. ${ }^{17}$ La construcción de este concepto se desarrolló en la medida que los griegos realizaron interpretaciones sobre la relación entre el hombre con su espacio y su posición en el universo. Los griegos, asimismo, establecieron oikoumene como un equivalente a Hélade, personificando su mundo a través de este concepto. Esta idea estuvo determinada por las diferentes interpretaciones que fueron evolucionando en la medida que el mundo heleno modificó sus paradigmas políticos, culturales, geográficos y religiosos a partir de la época helenística.

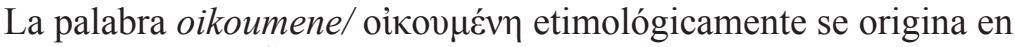
los sustantivos griegos oĩ oĩ̌ç/oikos define la "casa" u "hogar" abarcándola como un objeto desde el espacio físico en referencia a bienes muebles e inmuebles, y por otra parte, desde una forma social que se remite a la familia como institución u entidad política perteneciente a la pólis, en la cual se origina la reproducción material e ideológica de los ciudadanos de una comunidad. ${ }^{19}$ Por lo demás, la palabra $\gamma \tilde{\eta}$ señala a la tierra como un espacio físico. ${ }^{20}$ Finalmente el

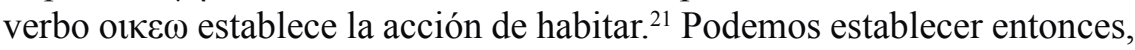
que al unir estas raíces existe una relación entre la tierra como un espacio para que el oikos lo pueda habitar, y la tierra en ese sentido se deberá

17 GEUS (2018).

$18 \quad$ Ibid.

19 MACDOWELL (1989:10); MIRÓN PÉREZ (2010:106; 2005:62-64).

20 BEEKES (2010:269-270).

21 Ibid., p. 1056. 
entender como el espacio habitado, la tierra habitada o el lugar habitado. ${ }^{22}$

Conjuntamente, debemos reconocer sin duda una evolución del concepto oikoumene desde el período clásico al helenístico. Por ejemplo, desde la perspectiva de Aristóteles la oikoumene estuvo integrada por los pueblos helenos y los no helenos. Demóstenes excluyó a todos los pueblos bárbaros, y finalmente, ya en plena época helenística este término se relacionó con los reinos helenísticos en un sentido político, en el cual al ejercer su dominio integraron estos territorios a la comunidad. Básicamente podemos establecer que oikoumene explicita la relación entre personas y entidades en un punto configurado para que estas lo habiten. ${ }^{23}$

El desarrollo geográfico de la antigüedad gozó de una particularidad: el encontrarse en una constante relación con las demás disciplinas del pensamiento griego. En vista de que no existió una división tajante entre ellas, el desarrollo geográfico dependió de la evolución de la techné para poder crear nuevos elementos que permitieron comprender el mundo. En ese sentido, la geografía tuvo como objeto el estudio el espacio griego o la oikoumene, y por ende, ante los avances geográficos la interpretación de este término también evolucionó.

El concepto de oikoumene se relacionó a la historiografía helenística en la medida que se preocupó del cambio de paradigma en la concepción del espacio griego. Podemos establecer que se desarrolló una transformación que se evidenció en los temas de estudio, que no sólo se centraron en los elementos locales, sino que la historiografía expresó interés en una historia total o universal.

El problema de la universalidad del mundo griego se remonta al período clásico, momento en que la construcción del relato historiográfico estuvo concentrado en la comunidad helena y su oposición a los pueblos barbaros ${ }^{24}$. La oikoumene, entonces, en su naturaleza cultural pasó a ser la equivalencia de Hélade, percibido como el espacio habitado por los helenos. Así, en la medida que evolucionó el relato historiográfico, al mismo tiempo se modificó el concepto de oikoumene para cada historiador.

22 MONLAU (1856: 251); GISINGER (1937:2123-2174).

23 ANEZIRI (2014:423-442).

24 MOMIGLIANO (1984:54). 
El primer proyecto de "historia universal" 25 se le atribuye a Éforo de Cime (380-330 a.C. $)^{26}$. Su obra se concentró en las diferentes entidades políticas distribuidas en las diferentes áreas geográficas del mundo habitado, elaborando un relato que incluyera todos los asuntos del mundo y no solamente los de algunos lugares. ${ }^{27}$

Esta modificación se fue estableciendo durante el desarrollo de la historiografía helenística, por lo que la escritura de la historia se orientó hacia un nuevo objeto de estudio, la oikoumene. La historia universal buscó narrar la historia contemporánea de la oikoumene considerando la evolución y expansión de la alteridad como base para cuestionar estos cambios. $^{28}$

\section{¿Qué es la oikoumene para Polibio?}

Esta pregunta es fundamental puesto que nos lleva a buscar la conexión entre dos marcos culturales que envuelven la figura de Polibio (Grecia y Roma), pero también nos induce a cuestionar la idea tradicional que indica que el mundo griego forjó la concepción del universo fundamentándola con elementos políticos, sino más bien, consideramos que se pensó simultáneamente con lo cultural.

Al resituar la postura de Polibio en el mundo griego, podemos establecer que su visión sobre los acontecimientos del mundo se desarrolló desde una óptica aquea, desde su perspectiva de intelectual griego que poseía amplios conocimientos en materia de guerra, política, economía y geografía. Cuando fue llevado como rehén a Roma, su carrera política se vio interrumpida, no obstante, a través de sus conocimientos buscó proyectar el ejercicio político truncado a través de la escritura de la historia en miras a retornar a Grecia en su debido momento. ${ }^{29}$

Uno de los objetivos de las Historias fue mostrar el mundo romano al mundo griego, en ese sentido podemos entender la transliteración y la traducción de elementos propios romanos para que los griegos pudieran entenderlos, esto tuvo por intención enseñar al público helénico con visión

25 Entendido como historia de la oikoumene.

26 Plb. V. 33.2.

27 CABALLERO (2007: 180-181).

28 INGLIS \& ROBERTSON (2005:108-110).

29 MORENO LEONI (2017:269). 
utilitarista el nuevo marco Mediterráneo.

Polibio construyó su concepción del universo sosteniéndose en dos factores: en primer lugar, la tradición geográfica precedente representada por los geógrafos Dicearco y Eratóstenes; y por otra parte, de su experiencia personal adquirida por sus viajes. El descubrimiento de occidente solamente fue posible gracias a su estadía en Roma y a la protección de los Escipiones.

La particularidad de las Historias de Polibio se encuentra en su carácter totalizante o universal por pretender narrar los acontecimientos relevantes y relacionados con la oikoumene. Es el mismo historiador quien explica su propósito.

"En las épocas anteriores a ésta los acontecimientos del mundo estaban como dispersos, porque cada una de las empresas estaba separada en la iniciativa de conquista, en los resultados que de ellas nacían y en otras circunstancias, así como en su localización. Pero a partir de esta época la historia se convierte en algo orgánico, los hechos de Italia y los de África se entrelazan con los de Asia y con los de Grecia, y todos comienzan a referirse a un único fin. Por esto hemos establecido en estos acontecimientos el principio de nuestra obra, porque en la guerra mencionada los romanos vencieron a los cartagineses, $\mathrm{y}$, convencidos de haber logrado ya lo más importante y principal de su proyecto de conquista universal, cobraron confianza entonces por primera vez para extender sus manos al resto: se trasladaron con sus tropas a Grecia y a los países de Asia"30

Los aportes geográficos para la construcción del espacio por parte de los romanos se distinguieron por ser de esencia predominantemente política, en el cual la conquista permitió tener un conocimiento preciso de los territorios. ${ }^{31}$ Polibio acompañando a Escipión Emiliano presenció muchos combates y saqueos de ciudades, como por ejemplo, la destrucción de Cartago.

Plb. 1.3.3-6. 
"Pues había leído que Escipión Emiliano, junto a Polibio de Megalópolis Arcadio, acompañados de treinta soldados, habían socavado una puerta de Cartago con ímpetu semejante." ${ }^{32}$

Las inferencias que podemos establecer es que Polibio logró obtener el conocimiento geográfico de occidente gracias a estos viajes, se desplazó desde la Galia hasta el norte de África, insertando sus vivencias en su relato y transmitiéndolos a su público, y al mismo tiempo reconociendo la nueva articulación política de los territorios mediterráneos dominados por los romanos. ${ }^{33}$

Otra perspectiva del concepto de oikoumene en Polibio se originó en torno a su concepción de cultura griega. Como hemos mencionado, durante el período clásico la definición de la identidad cultural estaba planteada por la relación centro/periferia, ubicando a lo bárbaro en los límites de la oikoumene. ${ }^{34}$ Durante la época helenística la difusión de los elementos culturales griegos se proyectaron hasta la India, esto se demuestra que con la fundación de ciudades por parte de Alejandro y la protección del helenismo a través de los reinos helenísticos, la cultura griega se halló en una estrecha relación con lo bárbaro ${ }^{35}$

Los fragmentos del libro XXXIV de las Historias nos han sido legados principalmente gracias a los trabajos de Estrabón, quien posiblemente realizó transcripciones literales del texto polibiano. ${ }^{36}$ Lo medular de esta digresión es la geografía como un recurso de importancia para el desarrollo de las Historias. Posiblemente en este libro Polibio se haya fijado el objetivo de realizar una descripción geográfica de la oikoumene, para así brindar a sus lectores un marco completo de los espacios en los cuales se desarrolló su obra. ${ }^{37}$

$32 \quad$ Plb. $39.19^{\mathrm{a}}$.

33 CRUZ ANDREOTTI (2002:44).

34 GÓMEZ ESPELOSÍN (2003:59).

35 Ibid, p. 72-74. Nosotros tomaremos para nuestro trabajo la idea de Cultura bárbara, en vista que muchos de los elementos que desarrollaron las sociedades orientales fueron fundamentales para el desarrollo de la cultura helena. 
La reconstrucción de este tratado fue realizado por Johann Schweighäuser durante el siglo XIX, quien los redactó manteniendo su forma íntegra en latín y articulándolos de la siguiente manera:1) introducción; 2) sobre los viajes de Ulises, con una especial referencia a Sicilia; 3) crítica a sus predecesores, muy en especial a Eratóstenes por su credibilidad del relato del viaje boreal de Piteas; 4) sobre los lusitanos; 5) sobre España en general; 6) sobre la Galia; 7) sobre Italia; 8) sobre Tracia, Macedonia y Grecia; 9) sobre Asia y finalmente 10) sobre Alejandría de Egipto. $^{38}$

Posteriormente Paul Pédech en 1956 realizó una nueva estructuración articulando estos fragmentos en tres principales ejes. En primer lugar, una topografía o descripción de los continentes y su posición en la esfera terrestre, en segundo lugar, una corografía o geografía descriptiva de Europa con una énfasis en occidente, y finalmente, en tercer lugar, una periodeia de África. ${ }^{39}$

Tomando en consideración la primera estructuración realizada por Schweighäuser, realizaremos una discusión e interpretación de algunos pasajes relevantes que se conservan del libro XXXIV, con la intención de situar la posición de las investigaciones de Polibio en el contexto helenístico.

La introducción al libro XXXIV se conserva principalmente gracias a Estrabón y Gémino ${ }^{40}$. En la primera sección se pueden distinguir dos elementos diferentes. El primero de estos guarda relación con el método historiográfico de Polibio, la búsqueda de la universalidad dentro de su relato y las críticas que realizó a autores precedentes. Y en una segunda parte, la construcción de una teoría sobre la tierra con base a las descripciones que elaboró a través de sus viajes.

Las Historias se identifican con la universalidad. Polibio no fue el

38 GARCÍA MORENO (2005:340-341).

39 Ibid.; WALBANK (1979:561 y ss).

40 Gémino fue un astrónomo que vivió durante el siglo I a.C. no se tiene mucho conocimiento sobre su vida, no obstante, se tiene certeza de solo un trabajo, Elementos de Astronomía, que plantea conceptos básicos en materia astronómica, geografía matemática y propone un sistema de Calendario. Su obra, además, una fuente importante para el conocimiento griego de la astronomía babilónica. TOOMER \& JONES (2016); También debemos destacar el aporte léxico de Gemino en esta especialidad. CALDERÓN DORDA (1998). 
primero en plantear una historia universal, ya que durante el siglo IV a.C. Éforo ya lo había hecho tratando los problemas históricos organizándolos de acuerdo a temáticas más que a un sentido cronológico. ${ }^{41}$ De igual manera, Polibio operó con la intención de realizar una descripción del mundo ${ }^{42}$, pero a su vez fue contrario a las ideas de Éforo, porque su interés se orientó al relato de una historia contemporánea, y no de historias pretéritas que abarcasen desde el origen. En ese sentido la obra de Polibio se conduce a un sistema causal en donde se debe tener necesariamente conocimiento de una primera causa que explique los acontecimientos. ${ }^{43}$ En uno de los pasajes del texto nos señala lo siguiente:

"Polibio dice que, por lo que se refiere a Grecia, Eudoxo hizo una buena exposición, y que Éforo la hizo muy buena, al tratar de la fundación de ciudades, genealogías, migraciones y establecimientos de colonias, «pero nosotros — añade - queremos describir la situación actual de las plazas e indicar las distancias, que es lo más esencial en la ciencia geográfica"44.

El fragmento anterior nos presenta una crítica realizada por Estrabón a la obra de Polibio. No obstante, la innovación a nivel historiográfico de Polibio se halla en el enfoque que le brindó su análisis geográfico, que no se interesó en la explicación de las fundaciones de las ciudades o las genealogías, sino más bien en los sucesos contemporáneos.

La medición de distancias que realizó Polibio fue posible a su carácter de estratega. El conocimiento de los trayectos que existen entre las ciudades fue una herramienta para planear maniobras de combate y tener un conocimiento real del espacio para el movimiento de los ejércitos. Polibio, en este sentido, invocó al principio de autopsía para poder construir el espacio.

La segunda parte de la introducción al libro XXXIV es la reproducción de las palabras de Polibio en la obra Elementos de Astronomía de Gemino.

$\begin{array}{ll}41 & \text { CABALLERO (2007:183). } \\ 42 & \text { WALBANK, (1977:572). } \\ 43 & \text { Plb. 1.5.2-5. } \\ 44 & \text { Plb. 34.1.3-5. } \\ 112 & \end{array}$


"El historiador Polibio ha compuesto un libro titulado Las partes del globo habitadas bajo el ecuador celeste. Éste está en la mitad de la zona tórrida. Asegura que esta región está habitada y que tiene un clima más templado que el que tienen los habitantes de los extremos de la zona tórrida. Por un lado, aporta las narraciones de los que han visto personalmente estas regiones y que dan testimonio de los hechos; por otro, arguye a base de la naturaleza de los movimientos del sol." ${ }^{\prime 4}$

El fragmento hace mención a una obra que aparentemente Polibio no escribió, ya que no se ha encontrado otra referencia en otros trabajos de la antigüedad. ${ }^{46}$ Sin embargo, el texto hace alusión a una reflexión sobre las partes del globo y sus habitantes. Gemino atribuyó a Polibio el aporte de narraciones de viajeros que han visto estas regiones, lo que permite plantear nuevamente la influencia de los estudios de Agatarcides de Cnido en la obra de Polibio. ${ }^{47}$ El uso de los relatos de viajes exploratorios fueron la base metodológica del relato polibiano. Esto fue planteado por el megapolitano como una solución metodológica ante la imposibilidad de la omnipresencia y la observación de todos los lugares del mundo. ${ }^{48}$

Ahora, por otra parte, Polibio realizó aportes con relación a la observación solar. Es de importancia este punto por que el uso de la geografía por parte de Polibio fue para buscar un marco espacial para el desarrollo de los acontecimientos de la oikoumene. En el libro III, ante el relato de la marcha de Aníbal a través de los Alpes, ofreció una distribución territorial tomando como referencia los puntos cardinales norte, sur, este y oeste ${ }^{49}$. Si bien este conocimiento no era una novedad, nos permite organizar la división del globo terrestre que presenta el libro XXXIV.

$45 \quad$ Plb. 34.1.7-9.

46 WALBANK (1979:573).

47 SANCHEZ LEÓN (1981:183-198).

48 Plb. 12.4c.4-5.

49 Plb. 3.37-38. 
"Si, como afirma Eratóstenes, la zona de debajo del ecuador es templada, opinión con la que está de acuerdo Polibio" 50

La introducción al libro XXXIV proyectó, además, un acercamiento a los elementos geográficos necesarios para el relato histórico, al mismo tiempo, de establecer algunos mecanismos que fueron tomados de autores anteriores, pero siempre orientando la digresión hacia las Historias y las necesidades que estas requieran.

La segunda parte del libro posiblemente se trató de un análisis de Polibio sobre los viajes de Ulises y la tradición homérica en un intento de buscar un espacio real a estos relatos. Debemos recordar que una de las principales características de la geografía del período helenístico es la crítica a la tradición homérica de astrónomos y geógrafos que abogaban más por una comprensión técnica del espacio, como es el caso de Eratóstenes e Hiparco. Sin embargo, la línea de Polibio se enmarcó en la de Aristarco y Catres de Malos, que sostuvieron una relación con las ideas de Homero. Sobre este último, debemos señalar que fue un componente fundamental de la educación griega durante la época helenística. ${ }^{51}$ Según un fragmento preservado en Estrabón dice que:

[Sobre los viajes de Ulises] "No es seguir el modo homérico apañar fantasmagorías totalmente infundadas. Es lógico que parezcan más creíbles aquellas mentiras en las que se mezcla algo de verdad, como afirma Polibio los errabundeos de Ulises." 52

Los textos de Homero han estado presentes constantemente en la concepción geográfica griega. Tomamos la lectura realizada por Frank Walbank en A historical commentary on Polybius, quien plantea el problema de Polibio al diferenciar qué lugares, países y viajes son parte de la ficción, y cuales podrían ubicarse en un mapa contemporáneo de la época polibiana. ${ }^{53}$ La Odisea es considerada una mezcla de las primeras experiencias de los

$50 \quad$ Plb. 34.1.16.

51 Ademas de Homero, son pilares de la educación clásica Eurípides, Menandro y Demóstenes. MARROU (2004:116-118).

Plb, 34.2.1-2. 
helenos por tierras de ultramar con los relatos fantasiosos originados en las fábulas y narraciones de la tradición literaria. ${ }^{54}$ Este inconveniente no es ajeno a Polibio en su intento de encontrar el territorio físico donde se desarrolló el periplo de Ulises.

Estrabón nos señala que Polibio tuvo una interpretación correcta de las navegaciones de Ulises. ${ }^{55}$ Esto queda demostrado con el caso de Éolo, quien en la obra de Homero es considerado el despensero de los vientos, a quien Polibio lo transportó a un plano concreto, en conjunto con el periplo de Ulises:

"Polibio no permite que Éolo sea presentado como algo mítico, ni tampoco todas las navegaciones de Ulises: afirma que hay algo de leyenda, como en la guerra narrada en la Ilíada" ${ }^{56}$

Si tomamos en cuenta el fragmento anterior, el relato de la Ilíada es parte también de la realidad y su existencia nos permite pensar a su vez que el catálogo de la naves disfrutó de la misma cualidad que las navegaciones de Ulises, por esto se presenta como como un suceso histórico. ${ }^{57}$

"Y si en Homero hay algo que no concuerde, la causa radica en un cambio, en ignorancia o en una licencia poética, que es una combinación de historia, disposición y mito. El fin que se propone la historia es la verdad, así, por ejemplo, en el Catálogo de las Naves el poeta nos explica lo que corresponde a cada ciudad: a una, la llama rocosa, de otra dice que está en los confines, a otra la califica de costera o de rica en palomas. [...] El fin del mito es el placer y el asombro. Inventárselo todo no es de fiar ni es propio de Homero". ${ }^{58}$

54 GÓMEZ ESPELOSÍN (2008:79).

$55 \quad$ Plb. 34.2.3.

56 Plb. 34.2.8-9.

57 La discusión sobre la naturaleza histórica o mitológica del catálogo de las naves lo podemos encontrar en GONZÁLEZ GARCÍA (200:57-72).

Plb. 34.4.1-4. 
El catálogo de las naves posee la particularidad geográfica que Polibio destacó y lo acercó al plano de lo real, haciendo la distinción entre lo mitológico y lo práctico. Es interesante, además, advertir que realizó una teorización en general de las ideas Homero, lo que permite construir una base de conocimiento geográfico con relación a los escritos homéricos para arremeter contra las ideas de Eratóstenes, crítico de Homero.

Polibio acercó a Homero a la realidad con propósito beneficioso al intentar ubicar concretamente los lugares que Ulises visitó durante su travesía. Existen dos fragmentos que nos permiten afirmar que Polibio supuso que estos viajes se realizaron en la zona de Sicilia.

"[Sobre los Viajes de Ulises] Estos errabundeos han sido, en general, colocados en Sicilia tanto por el poeta como por los demás escritores que tratan la historia local de Italia y de Sicilia." 59

Preliminarmente el fragmento nos señala que existen autores que en el pasado habían ubicado en Sicilia los viajes de Ulises. Ya habíamos advertido que para Polibio primó el principio de autopsía, lo que nos autoriza a establecer que en el libro XXXIV se escribieron las experiencias de Polibio recorriendo estos territorios. Esto se evidencia en una crítica que realizó a Eratóstenes ${ }^{60}$ ante la comparación de lo que ocurrió en el escollo de escila y la captura del pez espada.

Después de una descripción de carácter etnográfico sobre de los métodos por los cuales se capturan los peces espadas en los lugares cercanos a Sicilia, Polibio realizó una comparación con el método de pesca de los nativos cercanos del escollo de Escila ${ }^{61}$ presentado por Homero, por lo tanto, al presenciar estos lugares la relación con los pasajes del viaje de Ulises fueron innegable para el historiador.

Por último, podemos sostener que la aceptación de Polibio de los viajes expuestos en Homero, principalmente en la Odisea, responden a relacionar el relato del viaje como parte del método historiográfico, donde la exploración es fundamental para poder conocer el mundo y la mejor forma de obtener información de regiones que no se pueden visitar, pues

59 Plb. 34.2.9-10.

60 Quien realizó un trabajo crítico contra la obra de Homero.

61 Plb. 34.3.9-10. 
Polibio advierte que "no se puede ser testigo ocular de todos los lugares del mundo y de sus peculiaridades" ${ }^{62}$. Es posible que el relato Homérico garantice el uso de una supuesta fuente para establecer como real el relato y parece que Polibio buscaba promover a la Odisea como un texto confiable para el estudio geográfico.

\section{Hacia una nueva concepción del espacio griego}

La historiografía ha sido concluyente al establecer que la particularidad de las historias de Polibio se encuentra en su carácter universal, por este motivo, el argumento fundamental es que el mundo tendió a un único fin ${ }^{63}$. Esta disposición nos confirma que Polibio nos exhibe que esa era la aspiración de los romanos: la conquista del universo o de la oikoumene.

Polibio fue práctico al momento de instaurar el espacio geográfico, donde los libros, los testimonios y las descripciones a través de la autopsía estructuraron geográficamente la oikoumene. Pero, además, a través de la descripción física se puede construir una articulación de este concepto desde una perspectiva cultural, donde los elementos culturales del mundo griego fueron los fundamentos de este término.

Tradicionalmente se ha mencionado que los aportes geográficos para la construcción del espacio por parte de los romanos, se caracterizó por ser predominantemente de carácter político, en el cual la conquista facilitó el conocimiento preciso de los territorios. ${ }^{64}$

$62 \quad$ Plb. $12.4 c .2-5$.

63 Plb. 1.3.3-6: "En las épocas anteriores a ésta los acontecimientos del mundo estaban como dispersos, porque cada una de las empresas estaba separada en la iniciativa de conquista, en los resultados que de ellas nacían y en otras circunstancias, así como en su localización. Pero a partir de esta época la historia se convierte en algo orgánico, los hechos de Italia y los de África se entrelazan con los de Asia y con los de Grecia, y todos comienzan a referirse a un único fin. Por esto hemos establecido en estos acontecimientos el principio de nuestra obra, porque en la guerra mencionada los romanos vencieron a los cartagineses, $\mathrm{y}$, convencidos de haber logrado ya lo más importante y principal de su proyecto de conquista universal, cobraron confianza entonces por primera vez para extender sus manos al resto: se trasladaron con sus tropas a Grecia y a los países de Asia". 
Polibio inserto en esta nueva realidad construyó su imagen de oikoumene teniendo en consideración el dominio romano de casi todo el universo. Las conquistas posibilitan el conocimiento de un territorio, como por ejemplo, el caso de los Bematistas que recorrían junto a Alejandro para obtener comprensión de lo que se conquistó. Lo que hay acentuar es que Roma hasta la época de Polibio (siglo II a.C.) ejerció dominio por casi toda la cuenca del Mediterráneo, pero no sobre todos los rincones de la oikoumene.

En el caso del mundo griego el problema de la oikoumene se determinó directamente con lo cultural, puesto que la cultura griega definió sus fronteras. Los griegos definieron su espacio con relación a su identidad y por este motivo las regiones donde se expresó la cultura griega formaron parte del universo de Polibio.

Las conquistas de Alejandro proporcionaron elementos de cambio en la concepción del espacio griego en la medida que las fuentes, escritos y descripciones que se lograron recolectar en sus avanzadas sirvieron de base para autores posteriores. ${ }^{65}$ Uno de estos autores fue Eratóstenes y posteriormente Polibio.

En las Historias, Polibio afirma que Asia es conocida gracias a la expedición de Alejandro, y occidente para los griegos era ignorado. ${ }^{66}$ Polibio cuando visitó Alejandría, reconoció los componentes griegos de la ciudad y los contrapuso a los grupos que no eran griegos. Esta separación nos faculta interpretar que el interés de Polibio por Alejandría fue por su carácter griego, pese a la interacción de culturas. ${ }^{67}$

La contrariedad de Polibio y su concepción de la oikoumene se establecieron sobre el problema de alteridad con el mundo romano, y como estos pueden entrar a considerarse parte de la cultura helena. Tenemos que considerar que Polibio no abusó de la dualidad griego-bárbaro, pues en este nuevo contexto los romanos no se ajustaban estrictamente a la categoría peyorativa de bárbaros, pero tampoco era iguales a los griegos. ${ }^{68}$

65 GEHRKE (2016:97); GÓMEZ ESPELOSÍN (2003:72).

$66 \quad$ Plb. 3.59.3.

67 Plb. 39.14.4-5.

68 PELEGRÍN CAMPO (2004:61-62); También se debe considerar la situación de Polibio viviendo en Roma, y la visión de los romanos de forma más directa GONZALES RODRÍGUEZ (2005:144-147). 
Polibio exhibe a Roma como un modelo político y militar ejemplificador para el mundo griego. Por este motivo se justifica que el libro VI exponga la constitución romana, cuyo público son las póleis. Los griegos debieron aprender a conocer a Roma, en vista que a partir de ese momento se ubicó como la dominadora de Grecia. ${ }^{69}$

Probablemente Polibio aceptó que el conocimiento geográfico de occidente fue construido a través de las conquistas romanas. Esto puede que sea el motivo para realizar la crítica al viaje de Piteas, ya que si este fue verídico, su alcance incluyó lugares de los que Roma podía ejercer autoridad. Esto nos lleva a plantear que la construcción de la oikoumene estuvo en sintonía con la expansión del mundo romano.

Los griegos observaron a los romanos como un nuevo actor mediterráneo. Sobre la descripción de los territorios orientales sólo se conserva en las Historias un fragmento que entregó credibilidad y confianza al geógrafo Artemidoro. ${ }^{70}$ A esto se debe agregar que el cuadro geográfico que construyó Polibio para Oriente (hasta la India) también debe entrar en su concepción de oikoumene.

\section{Consideraciones finales}

Durante el siglo II d.C. se desarrolló un movimiento cultural identificado con el recuerdo de las imágenes del pasado griego, llamado Segunda Sofística, que utilizó la paideía como un componente unificador ante la autoridad romana. ${ }^{71}$ Durante este período el concepto oikoumene reaparece para designar el mundo dominado por los romanos remitiéndose a su categoría de superioridad cultural hacia otros pueblos. ${ }^{72}$ Roma logró conquistar casi todo el mundo conocido, situación que Polibio reconoció.

El concepto de oikoumene griega se construyó con los estudios de geografía en paralelo con otras disciplinas. Desde un análisis filológico hemos determinado que oikoumene significa "espacio habitado", y para poder comprender a cabalidad este concepto es necesario hacer una revisión sobre la historia de la cartografía y la importancia del mapa como un objeto gráfico de la oikoumene, que a través de su análisis es posible

69 MORENO LEONI (2013:85).

$70 \quad$ Plb. 34.13.

71 LAGOS ABURTO (2016:23).

72 HIDALGO DE LA VEGA (2008:48). 
tener conocimiento sobre la evolución en la concepción del mundo griego

La oikoumene finalmente se edificó principalmente en torno a dos conceptos: la techné y la cultura. En la medida que se lograron avances en el campo de la técnica, la concepción ecuménica también se modificó. Esto último se pudo conocer cuando se organizó una revisión sobre la evolución del pensamiento geográfico y sus representaciones a través del mapa. Por otra parte, el factor cultural del mismo modo fue determinante para este término que representa el espacio habitado por los helenos, y en este sentido mientras evolucionaron las ideas sobre civilidad y barbarie, la concepción de la oikoumene se transformó.

Polibio, un historiador del período helenístico que a través de sus Historias realizó una cantidad no menor de comentarios geográficos como un recurso para el desarrollo de su obra. Sus explicaciones geográficas bordean desde lo teórico hasta la descripción visual de los territorios que visitó, y su estadía en Roma fue determinante para que realizaran viajes a través del Mediterráneo y poder obtener certero conocimiento sobre los territorios occidentales, de los cuales el mundo griego no poseía una comprensión empírica, por lo que Polibio puede ser considerado como pionero para el desarrollo de las descripciones de occidente.

Para Polibio las conquistas romanas fueron de importancia para construir su conocimiento geográfico, y a través de la geografía logró elaborar su propia concepción de universo. La instrumentalización que realizó de la geografía nos faculta conocer cómo se configuró el espacio para el perfeccionamiento de su relato historiográfico, que tuvo un público principalmente griego. Podemos finalmente reconocer en las Historias una ambivalencia en la construcción de la oikoumene, que por una parte se originó por las conquistas y el establecimiento político romano, y por otra, desde una perspectiva cultural griega. 


\section{REFERENCIAS BIBLIOGRÁFICAS}

\section{Fuentes}

POLIBIO. (1981) Historias. Traducción de Manuel Balasch Recort, Madrid, Gredos.

\section{Bibliografía}

ANEZIRI, S. (2014). "Greek Strategies of Adaptation to the Roman World: The Case of the Contests", Mnemosyne, 67, 3, pp. 423-442.

BEEKES, R. S. P. (2010). Etymological Dictionary of Greek, Leiden, Brill. BETTALLI, M. (2009). Introduzione alla storiografia greca. Roma, Carocci.

BURSTEIN, S. (2016). "Greek Identity in the Hellenistic Period." en ZACHARIA, K. (ed.) Hellenisms: Culture, identity, and ethnicity from antiquity to modernity. London, Routledge, pp. 59-78.

CABALLERO. J. (2007). Inicios y desarrollo de la Historiografia griega: Mito, politica y propaganda. Madrid, Síntesis.

CALDERÓN DORDA, E. A. (1998) "La lengua científica de Gémino: La aportación léxica", Myrtia: Revista de filología clásica, 13, 1998, pp. 103-136.

CARBO, J; HIDALGO DE LA VEGA, M. (2008) "El ecumenismo romano en la época de Trajano: espacios de inclusión y exclusión" En Studia histórica. Historia antigua, 26, pp. 63-86.

CRUZ, ANDREOTTI, G. (2014). "La Geografía Griega como espacio político." En Geographia antiqua Vol.13, 2014, pp. 9-20.

--- (2009) "La naturaleza histórica de la "Geografía" de Estrabón", Euphrosyne: Revista de filología clássica, 37, pp. 131-144.

--- (2005) "Polibio y la geografía de la Península Ibérica: la construcción de un espacio político", en SANTOS YANGUAS J; TORREGARAY PAGOLA E. (eds.) Polibio y la Península Ibérica, Vitoria, Universidad del País Vasco.

--- (2004). "Geografía e historiografía clásica: el ejemplo de Polibio" Revista de historiografía (RevHisto), 1, pp. 60-70.

--- (2002) "La construcción de los espacios políticos Ibéricos entre los siglos III y I a.c.: algunas cuestiones metodológicas e históricas a partir de Polibio y Estrabón", en Cuadernos de prehistoria y arqueología, 28, 2002, pp. 35-54.

GARCÍA MORENO, L. A. (2005). "Polibio y la creación del estereotipo de lo hispano en la etnografía y la historiografía helenísticas", en SANTOS YANGUAS J; TORREGARAY PAGOLA E. (eds.) Polibio y la Península Ibérica, pp. 339-358. 
GEHRKE, H. (2016). "The "Revolution" of Alexander the Great: Old and New in the World's View", en BIANCHETTI, S; CATAUDELLA, M. R; GEHRKE, H. (eds.) Brill's companion to ancient geography. The inhabited world in Greek and Roman tradition, Leiden, Brill, pp. 78-97.

GEUS, K. (2018). "Oikoumene/Orbis Terrarum" en Dictionary of Classical Studies, Oxford University Press.

GISINGER, F. (1937). “Oikumene”, en Pauly-Wissowa XVII/2, 2123-2174.

GÓMEZ ESPELOSÍN, F. J. (2003). Los Griegos. Un legado universal, Madrid, Alianza Editorial.

GÓMEZ ESPELOSÍN, F.J; PÉREZ LAGACHA, A; VALLEJO GIRVÉS, M. (1994). Tierras fabulosas de la antigüedad, Alcalá, Servicio de Publicación Univ. de Alcalá.

GONZALES RODRÍGUEZ, M. C. (2005) "El bárbaro y lo bárbaro en la obra polibiana", en SANTOS YANGUAS J; TORREGARAY PAGOLA E. [eds.] Polibio y la Península Ibérica, Vitoria, Universidad del País Vasco, pp. 141-171.

GONZÁLEZ GARCÍA, F. J. (2001) "La geografía de los reinos de Argos y Micenas en el catálogo de las naves: ¿mito o historia?”, en LÓPEZ BARJA, P; REBOREDA MORILLO, S. (Eds.) Fronteras e identidad en el mundo griego antiguo. III. reunión de historiadores (Santiago de CompostelaTrasalba, 25-27 de septiembre de 2000), Santiago de Compostela, pp. 5772.

HIDALGO DE LA VEGA, M. (2008) "Ecumenismo romano: entre utopía y realidad": Studia histórica. Historia antigua, 26, pp. 47-62.

--- (2006) "Roma protectora del helenismo: el poder de la identidad" En DOMINGO PLÁCIDO (ed.), La construcción ideológica de la ciudadanía. Identidades culturales y sociedad en el mundo griego antiguo, Madrid: Editorial Complutense, pp. 423-448.

--- (2005). "Algunas reflexiones sobre los límites del oikoumene en el Imperio Romano." En Gerión. Revista de Historia Antigua, 23, 1, pp. 271-285.

INGLIS, D; ROBERTSON, R. (2005) "The Ecumenical Analytic", European Journal of Social Theory, 8, 2, pp. 99-122.

KAPLAN P. G (2018) "Early Greek Geography" en KEYSER, P. T; SCARBOROUGH, J; KAPLAN P. G (Eds.), Oxford Handbook of Science and Medicine in the Classical World, Oxford University Press, pp. 194214.

LAGOS ABURTO, L. (2016). El Helenismo en el siglo II d.C. La cultura griega a través de la Anabasis de Arriano de Nicomedia., Chillan, La Discusión S.A. 
MACDOWELL D. M. (1989) "The Oikos in Athenian Law", The Classical Quarterly, 39, 1, pp. 10-21.

MARROU, H. (2004) Historia de la educación en la Antigüedad, Madrid, Akal.

MEAGHER R. (1988). “Techne”, Perspecta, 24, pp. 159-164.

MEISTER, K. (1992). La storiografia greca: Dalle origini alla fine dell Ellenismo. Roma-Bari, Laterza.

MIRÓN PÉREZ, M. D. (2010) “El gobierno de la casa en Atenas clásica: género y poder en el "oikos"”, Studia Historica: Historia Antigua,18, pp. 103-117. --- (2005) "Oikos y oikonomia; El análisis de las unidades domésticas de producción y reproducción en el estudio de la Economía antigua", Gerión. Revista de Historia Antigua, 22, 1, pp. 61-79.

MOLINA, A. (2011) Geographica: Ciencia del espacio y tradición narrativa de Homero a Cosmas Indicopleustes. Murcia: Universidad de Murcia.

MOMIGLIANO, A. (1984) La historiografía griega. Barcelona, Crítica.

MONLAU, P. F. (1856) Diccionario etimológico de la lengue castellana precedido de unos rudimentos de etimología., Madrid, M. Rivadeneyra.

MORENO LEONI, Á. (2017). Entre Roma y el Mundo Griego: Memoria, Autorrepresentacion y didáctica del poder en las Historias de Polibio, Córdoba, Editorial Brujas.

--- (2013) "Interpretando el mundo romano: etnografía, público y cultura griega en las «Historias» de Polibio", Gerión. Revista de Historia Antigua, 30, pp. 63-90.

NICOLET, C. (1989). L'inventario del mondo. Geografia e politica alle origini dell'impero romano, Roma, Laterza.

--- (1982). Roma y la conquista del mundo mediterráneo, 264-27 a. de J.C., Barcelona, Labor.

---(2015). Space, geography, and politics in the early Roman Empire, Ann Arbor, University of Michigan Press.

PELEGRÍN CAMPO, J. (2004). "Tradición e innovación en la imagen polibiana del bárbaro", Studia Historica, Historia Antigua, 22, pp. 43-62

PLÁCIDO, D. (2008) "El ecumenismo romano desde la perspectiva de la historiografía griega: Dionisio de Halicarnaso" Studia historica. Historia antigua, 26, pp. 39-45;

--- (2008). "La ecúmene romana: espacios de integración y exclusión. Presentación" Studia historica. Historia antigua, 26, pp. 15-20

--- (1997). "La chóra y la oikouméne: la proyección geográfica del mundo colonial", Gerión. Revista de Historia Antigua, 15, pp. 79-86.

SANCHEZ LEÓN, M. L. (1981). "En torno a la transmisión de la obra de Agatárquides" Hispania antiqua, 11, pp. 183-198.

SCANLON, T. (2015). Greek historiography. Chichester, Wiley Blackwell. 
TABACHNICK, D. (2004). “Techne, Technology, and Tragedy”, Techné: Research in Philosophy and Technology, 7, 3, pp. 90-111.

TOOMER, G. J; JONES A. (2016) “Geminus" en Oxford Classical Dictionary, Oxford University Press.

TUPLIN, C. J; RIHLL T. E; WOLPERT, L. (2002). Science and Mathematics in Ancient Greek Culture, Oxford University Press.

WALBANK, F. W. (2006). Polybius, Rome and the Hellenistic World. Essays and Reflections, Cambridge University Press.

--- (1979). A historical commentary on Polybius. Vol. III. Commentary on Books XIX-XL, Oxford, Oxford et Clarendon Press. 